\title{
The Surgical Apgar Score in esophagectomy
}

\author{
Christopher F. Janowak, MD, Justin D. Blasberg, MD, Lauren Taylor, MD, James D. Maloney, MD, and \\ Ryan A. Macke, MD
}

\begin{abstract}
Objective: The Surgical Apgar Score is a validated prognostic tool that is based on select intraoperative variables (heart rate, mean arterial pressure, and blood loss). It has been shown to be a strong predictor of morbidity and mortality in a variety of surgical populations. Esophagectomy for malignancy represents a unique subset of patients at high risk for postoperative complications. This study assessed the ability of a modified esophagectomy Surgical Apgar Score (eSAS) to predict 30-day major morbidity.
\end{abstract}

Methods: A retrospective review included 168 patients who underwent elective esophagectomy for malignant disease at the University of Wisconsin from January 2009 through July 2013. Preoperative patient characteristics, intraoperative details, and short-term outcomes were recorded. Primary outcome was 30-day major morbidity. Univariate and multivariate analyses were performed to determine associations between predictive variables, eSAS, and major morbidity.

Results: Major morbidity occurred in 35\% of cases. Univariate analysis showed that eSAS of 6 or less was strongly associated with major morbidity (unadjusted odds ratio, $2.55 ; 95 \%$ confidence interval, $1.32-4.91 ; P=.005$ ). Other risk factors included transhiatal technique, body mass index less than 20 or greater than $35 \mathrm{~kg} / \mathrm{m}^{2}$, and history of diabetes mellitus. In multivariate analysis, eSAS of 6 or less remained a strong predictor of postoperative complications (adjusted odds ratio, 3.75; 95\% confidence interval, 1.70-8.26; $P=.001$ ).

Conclusions: The eSAS was strongly associated with 30-day major morbidity after esophagectomy. Prospective studies are needed to determine whether improved outcomes can be achieved with the eSAS for risk-stratified triage and postoperative care modification. (J Thorac Cardiovasc Surg 2015;150:806-12)

\begin{tabular}{|c|c|c|c|c|c|}
\hline \multicolumn{6}{|c|}{ Esophagectomy Surgical Apgar Score (eSAS) } \\
\hline & & 1 point & & 3 points & 4 points \\
\hline $\mathrm{EBL}(\mathrm{mL})$ & $>300$ & $201-300$ & $151-200$ & $\leq 150$ & \\
\hline Lowest MAP (mmHg) & $<40$ & $40-54$ & $55-69$ & 270 & \\
\hline Lowest HR (beats/minute) & & & & & $\leq 55$ \\
\hline \multicolumn{6}{|c|}{ Esophagectomy Surgical Apgar Score (eSAS). } \\
\hline \multicolumn{6}{|c|}{ Central Message } \\
\hline \multicolumn{6}{|c|}{$\begin{array}{l}\text { The esophagectomy Surgical Apgar Score, } \\
\text { derived from the intraoperative lowest heart } \\
\text { rate, lowest mean arterial pressure, and esti- } \\
\text { mated blood loss, is a strong predictor of major } \\
\text { morbidity. }\end{array}$} \\
\hline
\end{tabular}

\section{Perspective}

Intraoperative factors associated with adverse outcomes have not been extensively investigated for esophagectomy. The esophagectomy Surgical Apgar Score (eSAS) is a simple, objective score that is based on the intraoperative lowest HR, lowest MAP, and EBL. We found that an eSAS of 6 or less was strongly associated with major morbidity. Prospective studies are needed to determine whether riskstratified triage and modified care that are based on the eSAS will improve outcomes.

See Editorial Commentary page 813.
Despite advances in surgical technique and postoperative care, esophagectomy remains a high-risk procedure, with complications rates as high as $60 \%$ in select series. ${ }^{1-3}$ Numerous risk factors have been found to be associated with major morbidity and mortality after esophagectomy $^{3-5}$; however, previously reported risk models have been unreliable. ${ }^{6,7}$ These studies have primarily focused on preoperative risk assessment in an effort to improve outcomes through better patient selection and to facilitate risk-stratified comparative analyses for quality assessment.

From the Department of Surgery, University of Wisconsin School of Medicine and Public Health, Madison, Wis

Received for publication March 14, 2015; revisions received May 12, 2015; accepted for publication July 3, 2015; available ahead of print July 31, 2015.

Address for reprints: Ryan A. Macke, MD, H4/318 Clinical Sciences Center, 600 Highland Ave, Madison, WI 53792 (E-mail: macke@surgery.wisc.edu).

$0022-5223 / \$ 36.00$

Copyright (c) 2015 by The American Association for Thoracic Surgery

http://dx.doi.org/10.1016/j.jtcvs.2015.07.017
Although much attention has centered on preoperative risk factors in the esophagectomy population, there has been little investigation into risk stratification on the basis of intraoperative factors. In 2007, Gawande and colleagues ${ }^{8}$ developed a simple, objective scoring system to risk stratify general surgical and vascular surgical patients according to the operative course. Gawande and colleagues ${ }^{8}$ initially evaluated 28 intraoperative variables and found that the lowest heart rate (HR), lowest mean arterial blood pressure (MAP), and estimated blood loss (EBL) were independent predictors of 30-day major morbidity and mortality. These variables were incorporated into a 10-point scoring system, referred to as the Surgical Apgar Score (SAS). The SAS has now been externally validated in a number of large patient populations, ${ }^{9,10}$ for specific procedures, ${ }^{11-16}$ and for surgical specialties. ${ }^{17-19}$ These studies have demonstrated significant predictive value of the SAS for postoperative morbidity and mortality. 


\section{Abbreviations and Acronyms \\ $\mathrm{HR}=$ heart rate \\ MAP $=$ mean arterial blood pressure \\ EBL = estimated blood loss \\ SAS = Surgical Apgar Score \\ eSAS = esophagectomy Surgical Apgar Score \\ BMI = body mass index \\ ILE = Ivor Lewis esophagectomy \\ MKE = McKeown esophagectomy \\ NSQIP = National Surgical Quality Improvement Program \\ ICU = intensive care unit \\ $\mathrm{OR}=$ odds ratios \\ $\mathrm{CI}=$ confidence interval}

Intraoperative risk factors associated with adverse postoperative outcomes have not been extensively investigated in the esophagectomy population. A simple, objective scoring system that reassesses risk immediately after surgery might allow for more informed, risk-stratified triage of patients and for modified postoperative care. The purpose of this study was to assess the ability of a modified esophagectomy SAS (eSAS) to predict 30-day major morbidity after esophagectomy. Secondary outcomes investigated included prolonged stay and discharge disposition.

\section{MATERIALS AND METHODS \\ Patients}

A retrospective review of 172 patients who underwent esophagectomy at the University of Wisconsin Hospitals and Clinics (Madison, Wis) from January 2009 through July 2013 was performed. Patients were identified from an institutional database of major thoracic surgical procedures. Patients who underwent nonelective esophagectomy or underwent resection for benign disease were excluded $(n=4)$. Data collected included preoperative patient characteristics, intraoperative details, and short-term postoperative outcomes. Data not routinely collected in the institutional database were obtained by retrospective review of the medical record. Our institutional review board approved this study (IRB \#M-2009-1308). The need for individual consent was waived because of the retrospective nature of the analysis.

\section{Patient Characteristics}

Patient data, including age, sex, body mass index (BMI), American Society of Anesthesiologists score, smoking history, and comorbid disease, were recorded. Chronic comorbid conditions were recorded as composite variables for pulmonary disease (chronic obstructive or restrictive pulmonary disease), cardiovascular disease (coronary artery disease, congestive heart failure, peripheral vascular disease, or cerebrovascular disease), chronic kidney disease (baseline creatinine $>1.8 \mathrm{mg} / \mathrm{dL}$ or on hemodialysis), and diabetes mellitus. Administration of neoadjuvant chemotherapy or radiation therapy was also recorded.

\section{Intraoperative Data Collection}

Operative time, approach, and esophagectomy technique were recorded. Techniques included transhiatal esophagectomy, Ivor Lewis esophagectomy (ILE), and McKeown esophagectomy (MKE), which were performed through open, hybrid, or minimally invasive approaches. Hybrid approaches used a combination of minimally invasive and open approaches (laparoscopy and thoracotomy or laparotomy and thoracoscopy). Procedures that used only laparoscopic or thoracoscopic approaches were considered minimally invasive. Minimally invasive and hybrid approaches that required conversion were categorized as open procedures.

The methodology described by Regenbogen and colleagues ${ }^{9}$ was used to collect and assign points for the intraoperative lowest MAP and lowest HR. The ranges used to assign points for EBL in the original SAS scoring system were adjusted, with the EBL cutoff points based on quartile values of EBL in our patient cohort. Median EBL was $200 \mathrm{~mL}$ (range, $50-1500 \mathrm{~mL}$; interquartile range, 150-300 mL). Operative notes reporting "minimal" EBL were recorded as $50 \mathrm{~mL}$. Adjustment of the SAS EBL range has previously been described in validation studies for both gastrectomy and total cystectomy procedures. ${ }^{11,15}$ Details of the modified eSAS are shown in Box 1.

\section{Definition of Outcomes}

The primary outcome of our study was 30-day major morbidity. Similar to the original SAS study and subsequent validation studies, major complications were based on National Surgical Quality Improvement Program (NSQIP)-defined adverse events, including bleeding requiring at least 4 units of packed red blood cells within 72 hours of operation, cardiac arrest requiring cardiopulmonary resuscitation, myocardial infarction, deep venous thrombosis, pulmonary embolism, coma for at least 24 hours, stroke, acute renal failure, wound disruption, deep or organ-space surgical site infection, systemic inflammatory response syndrome, sepsis, septic shock, pneumonia, unplanned intubation, and ventilator use for at least 48 hours. ${ }^{20}$ Additional complications not meeting the NSQIP definitions for major complication were individually reviewed and evaluated according to the Clavien classification. ${ }^{21}$ Complications meeting the definition for Clavien class III complications (requiring surgical, endoscopic, or radiologic intervention) and class IV (requiring readmission to the intensive care unit [ICU] or considered life threatening) were also categorized as major morbidity. All patients with 30-day mortality had at least one major complication and therefore were recorded as having major morbidity. Esophagectomy-specific complications (anastomotic leak, gastric outlet obstruction, chylothorax, and recurrent laryngeal nerve injury) were also noted. All anastomotic leaks (clinically or radiographically detected) were recorded as deep or organ space surgical site infections, whether they were treated conservatively or with interventions such as percutaneous drainage, esophageal stenting, or reoperation. Other esophagectomy-specific complications were included the analysis only if they met the NSQIP definitions for major morbidity or Clavien class III or IV criteria. Prolonged stay was defined as a hospital stay longer than 10 days, which was determined by quartile values (median, 8 days; range 2-107 days; interquartile range, 7-10 days). Disposition status was defined as either home or nonhome discharge, excluding in-hospital deaths.

\section{Statistical Analysis}

To improve discriminative power, as well as ease of interpretation and clinical utility, preoperative and intraoperative continuous variables were grouped into clinically relevant categories, including age $(<50,50-59$, $60-69,70-79$, or $\geq 80$ days), BMI $(<20,20-24.9,25-29.9,30-34.9$, or $\geq 35 \mathrm{~kg} / \mathrm{m}^{2}$ ), operative time ( $<6$ hours or $\geq 6$ hours), and the focal variable of eSAS. The eSAS was assessed as a dichotomous variable for subgroup analysis. As in the study by Thorn and associates, ${ }^{22}$ the cutoff score with optimal accuracy for major morbidity was used to group patients into high-risk (below the cutoff) and low-risk (above the cutoff) eSAS cohorts. Accuracy was defined as the sum of true positives (number of patients below the cutoff score who had major complications) and true negatives (number of patients with a score above the cutoff who did not have major complications) divided by the total number of patients who underwent esophagectomy. Surgical approach was categorized as open, hybrid, or minimally invasive. Surgical technique was grouped as either transhiatal 
BOX 1. Modified esophagectomy Surgical Apgar Score

0 points 1 point 2 points 3 points 4 points

\begin{tabular}{lrccrr}
\hline EBL $(\mathrm{mL}) *$ & $>300$ & $201-300$ & $151-200$ & $\leq 150$ & \\
Lowest MAP (mm Hg) & $<40$ & $40-54$ & $55-69$ & $\geq 70$ & \\
Lowest HR (beats/min) & $>85$ & $76-85$ & $66-75$ & $56-65$ & $\leq 55$ \\
\hline
\end{tabular}

$E B L$, Estimated blood loss; $M A P$, mean arterial pressure; $H R$, heart rate. * Original Surgical Apgar Score EBL ranges: $>1000,601-1000,101-600$, and $\leq 100 \mathrm{~mL}$.

or transthoracic. Smoking history was categorized as current (smoking at the time of surgery or quit within month of surgery), former (smoking cessation $>1$ month before surgery), or never smokers. Data for all variables and outcomes were complete.

Associations between categoric variables and the outcomes of interest were assessed with the $\chi^{2}$ test. Unadjusted odds ratios (ORs) were determined by univariate logistic regression analysis and reported with a $95 \%$ confidence interval (CI). Multivariate logistic regression analysis included variables with a $P$ value less than .2 by univariate analysis. A backward likelihood ratio selection procedure was then performed to determine the predictors in the final multivariate model. Separate analysis was performed treating all appropriate variables in their continuous form and the eSAS as either a continuous or 4category variable (eSAS grouped as 0-4, 5-6, 7-8, and 9-10), as in the original SAS study. The discriminatory accuracy of the eSAS and multivariate models were assessed by determining the area under the receiver operating characteristics curve, reported as the $\mathrm{C}$ statistic. Calibration of the multivariate model was evaluated with the Hosmer-Lemeshow goodness-of-fit test. Statistical analyses were performed with the SPSS version 22 program (IBM Corporation, Armonk, NY). All tests were two-sided.

\section{RESULTS \\ Patients}

A total of 168 patients underwent elective esophagectomy by the thoracic surgery service at our institution for management of malignant disease. Seventy-three percent of the patients were male, and the mean age was $65 \pm 10$ years. Chronic comorbid disease was documented in $49 \%$ of patients $(\mathrm{n}=83)$. Mean operative time was $361 \pm 104$ minutes. Open approaches included transhiatal $(\mathrm{n}=49$; $29 \%)$, ILE $(n=19 ; 11 \%)$, and MKE $(n=1 ; 1 \%)$ esophagectomy techniques. Hybrid approaches included ILE $(\mathrm{n}=81$; $48 \%)$ and $\operatorname{MKE}(\mathrm{n}=2 ; 1 \%)$ techniques. Minimally invasive approaches were performed less often and included ILE $(\mathrm{n}=14 ; 8 \%)$ and $\operatorname{MKE}(\mathrm{n}=2 ; 1 \%)$ techniques.

\section{SAS Distribution and Cutoff}

The distribution of patients by eSAS is shown in Figure 1. The eSAS threshold to divide patients into high- and lowrisk groups was 6 or less, determined by the optimal accuracy $(0.61)$ at this cutoff point with reference to major morbidity. Eighty-two patients $(49 \%)$ received a score of 6 or less, comprising the high-risk cohort.

\section{Postoperative Outcomes}

Thirty-day operative mortality was $2.4 \%(n=4)$ of the patients, 3 of whom had an eSAS of 6 or less. Major postoperative complications occurred in $35 \%$ of patients $(\mathrm{n}=58)$. The most common NSQIP-defined complications were sepsis $(n=36 ; 21 \%)$, deep or organ space infection

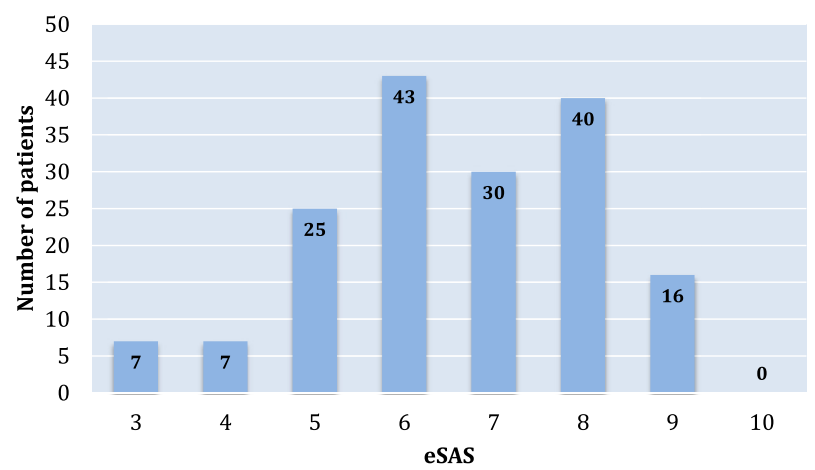

FIGURE 1. Distribution of patients by esophagectomy Surgical Apgar Score $(e S A S)$.

$(\mathrm{n}=31 ; 18 \%)$, and pneumonia $(\mathrm{n}=23 ; 14 \%)$. Other Clavien class III or IV complications not otherwise meeting the NSQIP definition for major morbidity occurred in $14 \%$ of patients $(\mathrm{n}=23)$. Esophagectomy-specific complications included anastomotic leak $(\mathrm{n}=26 ; 15 \%)$, chylothorax $(\mathrm{n}=5 ; 3 \%)$, gastric outlet obstruction $(\mathrm{n}=2 ; 1 \%)$, and recurrent laryngeal nerve injury $(\mathrm{n}=2 ; 1 \%)$.

Univariate analysis. Results of the univariate analysis for the outcome of major morbidity are shown in Table 1 for preoperative variables and Table 2 for intraoperative variables. An eSAS of 6 or less was strongly associated with major morbidity (unadjusted OR, 2.55 ; 95\% CI, 1.32$4.91 ; P=.005)$. The eSAS was also strongly associated with major morbidity when treated as the alternative 4-category variable, grouped as eSAS of 0 to 4,5 or 6,7 or 8 , and 9 or $10(P=.002)$. The eSAS did not have a linear association with postoperative complications when analyzed as a continuous variable $(P=.095)$, likely due to the small number of patients at the score extremes (Figure 1) and the limited power of our study.

Other variables associated with postoperative morbidity included transhiatal technique (OR, 2.12; 95\% CI, 1.07$4.21 ; P=.034)$, BMI less than $20 \mathrm{~kg} / \mathrm{m}^{2}$ (OR, 7.83; $95 \% \mathrm{CI}, 1.85-33.16 ; P=.005)$, BMI greater than $35 \mathrm{~kg} /$ $\mathrm{m}^{2}$ (OR, 5.28; 95\% CI, 1.54-18.12; $P=.008$ ), and history of diabetes mellitus (OR, 2.30; 95\% CI, 1.09-4.88; $P=.030)$. The individual variables making up the eSAS were not independently associated with postoperative morbidity when analyzed in a continuous or categoric fashion according to the eSAS point system.

Complications occurring in 5 or more patients are shown in Table 3. All complications occurred more frequently in the high-risk group; however, only the differences in unplanned intubation $(5 \%$ vs $16 \% ; P=.026)$ and septic shock $(1 \%$ vs $9 \% ; P=.031)$ were statistically significant. Patients in the high-risk cohort were more likely to have a prolonged stay ( $>10$ days) compared with those with a score greater than 6 ( $48.8 \%$ vs $32.6 \% ; P=.032)$; however, eSAS was not associated with nonhome discharge (eSAS $\leq 6$, $14.6 \%$ vs eSAS $>6,9.3 \% ; P=.286$ ). 
TABLE 1. Preoperative patient characteristics and results of univariate analysis

\begin{tabular}{|c|c|c|c|c|}
\hline Patient characteristics & $\mathbf{N}(\%)$ & Morbidity ( $\%$ of subgroup) & Unadjusted OR (95\% CI) & $P$ value \\
\hline Total & $168(100 \%)$ & $58(35 \%)$ & & \\
\hline \multicolumn{5}{|l|}{ Sex } \\
\hline Male & $122(73 \%)$ & $45(37 \%)$ & Reference & \\
\hline Female & $46(27 \%)$ & $13(28 \%)$ & $0.68(0.32-1.41)$ & 296 \\
\hline Age (y) & & & & .952 \\
\hline$<50$ & $9(5 \%)$ & $3(33 \%)$ & Reference & \\
\hline $50-59$ & $47(28 \%)$ & $17(36 \%)$ & $1.13(0.25-5.12)$ & .871 \\
\hline $60-69$ & $50(30 \%)$ & $15(30 \%)$ & $0.86(0.19-3.89)$ & .842 \\
\hline $70-79$ & $51(30 \%)$ & $19(37 \%)$ & $1.19(0.27-5.31)$ & .822 \\
\hline$>80$ & $11(7 \%)$ & $4(36 \%)$ & $1.14(0.18-7.28)$ & .888 \\
\hline \multicolumn{5}{|l|}{ ASA class } \\
\hline II & $62(37 \%)$ & $17(27 \%)$ & Reference & \\
\hline III-IV & $106(63 \%)$ & $41(39 \%)$ & $1.67(0.85-3.30)$ & .140 \\
\hline BMI $\left(\mathrm{kg} / \mathrm{m}^{2}\right)$ & & & & .002 \\
\hline$<20$ & $11(7 \%)$ & $8(73 \%)$ & $7.83(1.85-33.16)$ & .005 \\
\hline $20-24.9$ & $47(28 \%)$ & $12(26 \%)$ & $1.08(0.42-2.38)$ & .987 \\
\hline $25.0-29.9$ & $63(38 \%)$ & $16(25 \%)$ & Reference & \\
\hline $30-34.9$ & $33(20 \%)$ & $13(39 \%)$ & $1.91(0.78-4.70)$ & .159 \\
\hline$>35$ & $14(8 \%)$ & $9(64 \%)$ & $5.28(1.54-18.12)$ & .008 \\
\hline Smoking & & & & .171 \\
\hline Current & $19(11 \%)$ & $8(42 \%)$ & $2.18(0.91-5.20)$ & .114 \\
\hline Former & $112(67 \%)$ & $42(38 \%)$ & $2.64(0.79-8.76)$ & .080 \\
\hline Never & $37(22 \%)$ & $8(22 \%)$ & Reference & \\
\hline \multicolumn{5}{|l|}{ Comorbid disease } \\
\hline \multicolumn{5}{|l|}{ Pulmonary } \\
\hline History & $23(14 \%)$ & $10(43 \%)$ & $1.55(0.64-3.8)$ & .333 \\
\hline None & $145(86 \%)$ & $48(33 \%)$ & Reference & \\
\hline \multicolumn{5}{|l|}{ Cardiovascular } \\
\hline History & $58(35 \%)$ & $23(40 \%)$ & $1.41(0.73-2.73)$ & .311 \\
\hline None & $110(65 \%)$ & $35(32 \%)$ & Reference & \\
\hline \multicolumn{5}{|l|}{ Chronic renal disease } \\
\hline History & $17(10 \%)$ & $8(47 \%)$ & $1.80(0.65-4.93)$ & .256 \\
\hline None & $151(90 \%)$ & $50(33 \%)$ & Reference & \\
\hline \multicolumn{5}{|l|}{ Diabetes mellitus } \\
\hline History & $36(21 \%)$ & $18(50 \%)$ & $2.30(1.09-4.88)$ & .030 \\
\hline None & $132(79 \%)$ & $40(30 \%)$ & Reference & \\
\hline \multicolumn{5}{|l|}{ Neoadjuvant CRT } \\
\hline History & $93(55 \%)$ & $30(32 \%)$ & $0.80(0.42-1.51)$ & .492 \\
\hline None & $75(45 \%)$ & $28(37 \%)$ & Reference & \\
\hline
\end{tabular}

OR, Odds ratio; $C I$, confidence interval; $A S A$, American Society of Anesthesiologists; $B M I$, body mass index; $C R T$, chemoradiation therapy.

Of the preoperative and intraoperative variables, only prolonged operative time ( $>6$ hours) was associated with an eSAS of 6 or less $(48.8 \%$ vs $32.6 \% ; P=.032)$. There was no difference in the proportion of patients receiving an eSAS of 6 or less according to transhiatal versus transthoracic esophagectomy technique $(55 \%$ vs $46 \%$; $P=.313$ ) or according to operative approach (open, $57 \%$; hybrid, $47 \%$; minimally invasive, $56 \% ; P=.133$ ).

Overall, $31.5 \%$ of patients were admitted to the ICU immediately after surgery (eSAS $>6, \mathrm{n}=24 ; 27.9 \%$; eSAS $\leq 6, \mathrm{n}=29 ; 35.4 \%$ ). In the low-risk group (eSAS $>6$ ), $16.6 \%$ of patients admitted to the ICU and $27.4 \%$ admitted to a non-ICU setting had major complications
$(P=.775)$. Complications occurred less often when patients in the high-risk group were admitted to the ICU versus a non-ICU setting $(31.0 \%$ vs $52.8 \% ; P=.067)$, although this difference was not statistically significant.

Multivariate analysis. Independent variables with a $P$ value of less than .2 by univariate analysis were included in the multivariate analysis. A backward likelihood ratio selection procedure was performed, and variables remaining in the logistic regression model included American Society of Anesthesiologists score, BMI, diabetes mellitus, surgical technique, and eSAS. After multivariate analysis, eSAS of 6 or less (adjusted OR, 3.75; 95\% CI, 1.70-8.26; $P=.001$ ) remained a strong predictor of major morbidity. American 
TABLE 2. Intraoperative patient characteristics and results of univariate analysis

\begin{tabular}{|c|c|c|c|c|}
\hline $\begin{array}{c}\text { Patient } \\
\text { characteristics }\end{array}$ & $\mathbf{N}(\%)$ & $\begin{array}{r}\text { Morbidity (\% } \\
\text { of subgroup) }\end{array}$ & $\begin{array}{c}\text { Unadjusted } \\
\text { OR }(95 \% \text { CI })\end{array}$ & $\begin{array}{c}P \\
\text { value }\end{array}$ \\
\hline Total & $168(100 \%)$ & $58(35 \%)$ & & \\
\hline \multicolumn{4}{|l|}{ Surgical approach } & .049 \\
\hline Open & $69(41 \%)$ & $31(45 \%)$ & $3.54(0.92-13.53)$ & .065 \\
\hline Hybrid & $83(49 \%)$ & $24(29 \%)$ & $1.76(0.46-6.75)$ & .408 \\
\hline MIE & $16(10 \%)$ & $3(19 \%)$ & Reference & \\
\hline \multicolumn{5}{|l|}{ Surgical technique } \\
\hline Transthoracic & $119(71 \%)$ & $35(29 \%)$ & Reference & \\
\hline Transhiatal & $49(29 \%)$ & $23(47 \%)$ & $2.12(1.07-4.21)$ & .034 \\
\hline \multicolumn{5}{|l|}{ Operative time } \\
\hline$<6 \mathrm{~h}$ & $100(60 \%)$ & $34(34 \%)$ & Reference & \\
\hline$>6 \mathrm{~h}$ & $68(40 \%)$ & $24(35 \%)$ & $1.06(0.55-2.02)$ & .863 \\
\hline \multicolumn{5}{|l|}{ eSAS } \\
\hline$\leq 6$ & $82(49 \%)$ & $37(45 \%)$ & $2.55(1.32-4.91)$ & .005 \\
\hline$>6$ & $86(51 \%)$ & $21(24 \%)$ & Reference & \\
\hline
\end{tabular}

$O R$, Odds ratio; $C I$, confidence interval; $M I E$, minimally invasive esophagectomy; eSAS, esophagectomy Surgical Apgar Score.

Society of Anesthesiologists class III or IV, BMI less than 20 or more than $35 \mathrm{~kg} / \mathrm{m}^{2}$, and history of diabetes mellitus were other predictors of major morbidity (Table 4). The eSAS was also associated with postoperative complications when analyzed as a continuous variable $(P=.025)$ and as a 4-group categoric variable $(P=.001)$ in the multivariate models.

The high-risk versus low-risk eSAS multivariate model demonstrated good discriminatory accuracy (C statistic, $0.786 ; 95 \% \mathrm{CI}, 0.72-0.86)$ with adequate model calibration $(P=.887)$. Multivariate analysis performed in the same fashion without the eSAS also performed well, with a $\mathrm{C}$ statistic of 0.752 (95\% CI, 0.68-0.83) and adequate calibration $(P=.532)$. The discriminatory accuracy of the eSAS alone was only fair, with a $\mathrm{C}$ statistic of $0.614(95 \% \mathrm{CI}, 0.53-0.70)$.

TABLE 3. Specific complications and association with eSAS score

\begin{tabular}{lccc}
\hline \multicolumn{1}{c}{ Complication* } & $\begin{array}{c}\text { Low-risk } \\
(\mathbf{e S A S}>\mathbf{6} ; \\
\mathbf{n}=\mathbf{8 6})\end{array}$ & $\begin{array}{c}\text { High risk } \\
(\mathbf{e S A S} \leq \mathbf{6 ;} \\
\mathbf{n}=\mathbf{8 2})\end{array}$ & $\begin{array}{c}\boldsymbol{P} \\
\text { value }\end{array}$ \\
\hline Reintubation & $4(5 \%)$ & $13(16 \%)$ & .026 \\
Prolonged ventilation & $6(7 \%)$ & $13(16 \%)$ & .089 \\
Pneumonia & $8(9 \%)$ & $15(18 \%)$ & .112 \\
Deep wound or organ & $11(13 \%)$ & $19(23 \%)$ & .107 \\
$\quad$ space SSI & $14(16 \%)$ & $22(27 \%)$ & .132 \\
Sepsis & $1(1 \%)$ & $7(9 \%)$ & .031 \\
Septic shock & $9(10 \%)$ & $14(17 \%)$ & .261 \\
Other Clavien class III or IV & $10(12 \%)$ & $16(20 \%)$ & .201 \\
Anastomotic leak $\dagger$ & $1(1 \%)$ & $4(5 \%)$ & .202 \\
Chylothorax $\ddagger$ &
\end{tabular}

Data represent numbers and percentages of patients. SSI, Surgical site infection; eSAS, esophagectomy Surgical Apgar Score. *Some patients had more than 1 complication. $\dagger$ All anastomotic leaks were categorized as deep wound surgical site infections, in addition to other associated complications if they occurred (eg, sepsis). $\ddagger$ Requiring percutaneous intervention or reoperation (categorized as other Clavien class III for analysis).
TABLE 4. Variables included in final multivariate model and adjusted odds ratios

\begin{tabular}{lcc}
\hline \multicolumn{1}{c}{ Variable } & Adjusted OR $(\mathbf{9 5} \%$ CI) & $\boldsymbol{P}$ value \\
\hline Transhiatal technique & $2.14(0.98-2.68)$ & .058 \\
ASA class III-IV & $2.57(1.13-5.84)$ & .024 \\
BMI $<20 \mathrm{~kg} / \mathrm{m}^{2}$ & $10.34(2.13-50.35)$ & .004 \\
BMI $>35 \mathrm{~kg} / \mathrm{m}^{2}$ & $4.34(1.15-16.36)$ & .030 \\
Diabetes mellitus & $2.64(1.12-5.84)$ & .024 \\
eSAS $\leq 6$ & $3.75(1.70-8.26)$ & .001 \\
\hline
\end{tabular}

$O R$, Odds ratio; $C I$, confidence interval; $A S A$, American Society of Anesthesiologists; $B M I$, body mass index; $e S A S$, esophagectomy Surgical Apgar Score.

\section{DISCUSSION}

This study demonstrates that the eSAS is an independent predictor of 30-day major morbidity after esophagectomy. These findings are consistent with previous SAS validation studies in large patient populations, ${ }^{9,10}$ other procedures, ${ }^{11-16}$ and other surgical specialties. ${ }^{17-19}$ The eSAS was a strong predictor of postoperative complications when treated as a dichotomized, categoric, or continuous variable. It is our opinion that the eSAS is more clinically useful when the score is used to stratify patients into high-risk (eSAS $\leq 6)$ and low-risk groups (eSAS >6). To our knowledge, this is the first study validating the SAS exclusively in an esophagectomy population.

It is clear that there is no holy grail for predicting postoperative complications in this complex patient population. A number of predictive models for postoperative morbidity and mortality after esophagectomy have been developed, focusing primarily on fixed, preoperative risk factors $^{23-29}$; however, attempts to validate these risk stratification tools externally have demonstrated limited clinical applicability. ${ }^{7,30-32}$ A recent systematic review of 20 clinical prediction models for mortality after esophagectomy concluded that these models were unreliable because of poor discrimination and inadequate validation. ${ }^{6}$ One potential explanation for this suboptimal performance could be the failure to take into consideration patients' operative course. The eSAS is inherently different from these other prediction models in that risk assessment is done at a completely different point in time, taking into account events that occur during the critical hours in the operating room. The information gained by the eSAS can be used in combination with what is known about a patient's preoperative risk for better anticipation of adverse postoperative outcomes.

The SAS is a simple tool that facilitates an objective summary of patients' intraoperative course and may be ideally suited to a complex procedure like esophagectomy. It is not surprising that EBL, MAP, and HR were associated with postoperative outcomes in the original SAS study, as others have demonstrated persistent tachycardia and hypotension are strongly associated with adverse postoperative outcomes. ${ }^{8,33}$ Although intraoperative risk factors have 
not been extensively investigated in the esophagectomy population, it is generally accepted that a state of poor perfusion negatively impacts postoperative outcomes because of the tenuous blood supply of the gastric conduit. ${ }^{34}$ Inadequate systemic perfusion may occur as a result of significant EBL, whereas tachycardia and hypotension are expected physiologic responses to a hypoperfused state. A low eSAS score should therefore reflect intraoperative hemodynamic instability and an increased risk of inadequate perfusion. Interestingly, we did not find that the individual variables making up the eSAS were independently associated with major morbidity. From a clinical standpoint, it makes sense that patients with the combination of significant EBL with resulting tachycardia and hypotension (therefore receiving a low eSAS) would have a greater risk of postoperative complications. It is this unique combination of lowest HR, lowest MAP, and EBL that has repeatedly been shown to be associated strongly with adverse postoperative outcomes.

There are a number of potential ways in which the eSAS can be applied to clinical practice. Reassessing risk immediately after surgery with the eSAS allows for a patient's condition to be conveyed to other care providers in a consistent manner, rather than reporting a subjective assessment of the operation (eg, a "smooth" or "rocky" course). The simply calculated eSAS may also allow for modification of postoperative care. Rather than the decision to admit a patient to the ICU being based on a subjective assessment of the operative course, the decision can instead be based on easily obtained objective data. High-risk patients may be admitted to an ICU, and awareness of early signs of slowly progressing potential complications, such as infection and anastomotic leak, may be heightened. Appropriate risk-based triage also has the potential to decrease complications, resource utilization, and hospital cost by using risk-modified treatment strategies for high-risk patients. In retrospect, without the eSAS to guide our decision making, we discovered that $27.9 \%$ of the patients $(n=24)$ who would have been considered low risk (eSAS $>6$ ) were admitted to the ICU and that only $16.6 \%$ of these patients $(n=4)$ actually had major morbidity. No difference was noted in the complication rate when low-risk patients were admitted to an ICU or non-ICU setting. These patients therefore could likely have been admitted to a non-ICU setting, saving vital nursing resources and decreasing hospital cost. On the other hand, more than half the patients with an eSAS of 6 or less admitted to a non-ICU setting had major complications versus a lower proportion of those admitted to the ICU $(52.8 \%$ vs $31.0 \% ; P=.067)$. Although it is impossible to determine in a retrospective manner, this suggests that postoperative ICU-level care could potentially decrease complications in high-risk patients undergoing esophagectomy.

As suggested by Gawande ${ }^{8}$ and Regenbogen, ${ }^{9}$ the eSAS may also be used to facilitate quality assessment and improvement. The eSAS may be used to stimulate review of cases with lower scores to determine where improvements can be made. Surgeons with lower-scoring patients who are consistently found to have greater EBL may consider a change in surgical technique or approach. Cases with persistent tachycardia may be examined to determine whether this was due to failure to administer preoperative $\beta$-blockade, inadequate volume resuscitation during the procedure, or a myriad of other potential causes. A focused review of cases with persistent or significant hypotension may discover that specific antihypertensive medications, such as angiotensin-converting enzyme inhibitors, were not appropriately discontinued before surgery or that inadequate volume resuscitation was provided. The eSAS provides a simple and efficient way to identify these cases for root cause analysis.

A few limitations of this study should be taken into consideration when interpreting these results. This is a singleinstitution study with a relatively small sample size, limiting the power of our study. Further validation of the eSAS with larger data sets is needed, with additional assessment of the eSAS as a predictor for mortality (there were too few deaths to assess this adequately in our patient cohort). The definitions used to report short-term outcomes such as major morbidity have been shown to be heterogeneous and inconsistently reported in the esophagectomy literature. ${ }^{35}$ We chose to use the well-known NSQIP and Clavien classification definitions for postoperative adverse events to remain consistent with previous studies validating the SAS; however, application of the eSAS to other populations could yield different results simply because of the definition of major morbidity. As other authors have noted ${ }^{15,17}$ one of the potential weakness of the SAS is the use of EBL for calculation of the score, which can be inaccurate and may differ significantly with regard to the procedure performed as well as operative technique. We attempted to correct for this with the modified EBL point assignments to calculate the eSAS. Finally, the eSAS can be altered in a variety of ways, such as more aggressive or restricted use of vasoactive medications, preoperative $\beta$ blockade, and fluid management. On the basis of the presented data and previous SAS validation studies, however, it appears that regardless of the way in which a patient is managed intraoperatively, the combination of lowest HR, lowest MAP, and EBL are strongly associated with adverse outcomes. Further investigation is needed to determine whether modifications in management aimed at optimizing a patient's eSAS will result in improved postoperative outcomes.

In conclusion, we have shown that the eSAS is strongly associated with 30-day major morbidity after esophagectomy. Odds of major morbidity were approximately 2.5 times greater for patients with an eSAS of 6 or less when used as an independent predictor and nearly 4 times greater when other important preoperative and intraoperative 
factors were taken into account. The eSAS is simple to calculate, is composed of only 3 variables that are universally recorded during surgery, and can be used to convey information about the operative course in a consistent, objective manner. The eSAS may be clinically useful in terms of quality assessment and improvement by facilitating identification of patients who have had a suboptimal operative course. Prospective studies with routine recording of eSAS are needed to determine whether risk-stratified triage of patients and modification of postoperative care according to the eSAS result in decreased postoperative major morbidity, mortality, resource use, and hospital cost.

\section{Conflict of Interest Statement}

Authors have nothing to disclose with regard to commercial support.

We thank Glen Leverson, BA, PhD, for his assistance with the development of the statistical methodology and analysis.

\section{References}

1. Dimick JB, Cattaneo SM, Lipsett PA, Pronovost PJ, Heitmiller RF. Hospital volume is related to clinical and economic outcomes of esophageal resection in Maryland. Ann Thorac Surg. 2001;72:334-9; discussion 339-41.

2. Kuo EY, Chang Y, Wright CD. Impact of hospital volume on clinical and economic outcomes for esophagectomy. Ann Thorac Surg. 2001;72:1118-24.

3. Birkmeyer JD, Siewers AE, Finlayson EV, Stukel TA, Lucas FL, Batista I, et al. Hospital volume and surgical mortality in the United states. N Engl J Med. 2002; 346:1128-37.

4. Wright CD, Kucharczuk JC, O'Brien SM, Grab JD, Allen MS, Society of Thoracic Surgeons General Thoracic Surgery Database. Predictors of major morbidity and mortality after esophagectomy for esophageal cancer: a Society of Thoracic Surgeons General Thoracic Surgery Database risk adjustment model. J Thorac Cardiovasc Surg. 2009;137:587-95. Erratum in: J Thorac Cardiovasc Surg. 2009; 137:1581

5. Grotenhuis BA, Wijnhoven BP, Grüne F, van Bommel J, Tilanus HW, van Lanschot JJ. Preoperative risk assessment and prevention of complications in patients with esophageal cancer. J Surg Oncol. 2010;101:270-8.

6. Warnell I, Chincholkar M, Eccles M. Predicting perioperative mortality after oesophagectomy: a systematic review of performance methods of multivariate models. Br J Anaesth. 2015;114:32-43.

7. Zingg U, Langton C, Addison B, Wijnhoven BP, Forberger J, Thompson SK, et al. Risk prediction scores for postoperative mortality after esophagectomy: validation of different models. J Gastrointest Surg. 2009;13:611-8.

8. Gawande AA, Kwaan MR, Regenbogen SE, Lipsitz SR, Zinner MJ. An Apgar score for surgery. J Am Coll Surg. 2007;204:201-8.

9. Regenbogen SE, Ehrenfeld JM, Lipsitz SR, Greenberg CC, Hutter MM, Gawande AA. Utility of the surgical Apgar score: validation in 4119 patients. Arch Surg. 2009;144:30-6; discussion 37.

10. Haynes AB, Regenbogen SE, Weiser TG, Lipsitz SR, Dziekan G, Berry WR, et al. Surgical outcome measurement for a global patient population: validation of the surgical Apgar score in 8 countries. Surgery. 2011;149:519-24.

11. Prasad SM, Ferreria M, Berry AM, Lipsitz SR, Richie JP, Gawande AA, et al. Surgical Apgar outcome score: perioperative risk assessment for radical cystectomy. J Urol. 2009;181:1046-52; discussion 1052-3.

12. Wuerz TH, Regenbogen SE, Ehrenfeld JM, Malchau H, Rubash HE, Gawande AA, et al. The surgical Apgar score in hip and knee arthroplasty. Clin Orthop Relat Res. 2011;469:1119-26.

13. Regenbogen SE, Bordeianou L, Hutter MM, Gawande AA. The intraoperative Surgical Apgar Score predicts postdischarge complications after colon and rectal resection. Surgery. 2010;148:559-66.
14. La Torre M, Ramacciato G, Nigri G, Balducci G, Cavallini M, Rossi M, et al. Post-operative morbidity and mortality in pancreatic surgery. The role of surgical Apgar score. Pancreatology. 2013;13:175-9.

15. Miki Y, Tokunaga M, Tanizawa Y, Bando E, Kawamura T, Terashima M. Perioperative risk assessment for gastrectomy by surgical Apgar score. Ann Surg Oncol. 2014;21:2601-7.

16. Zighelboim I, Kizer N, Taylor NP, Case AS, Gao F, Thaker PH, et al. "Surgical Apgar Score" predicts postoperative complications after cytoreduction for advanced ovarian cancer. Gynecol Oncol. 2010;116:370-3.

17. Reynolds PQ, Sanders NW, Schildcrout JS, Mercaldo ND, St Jacques PJ. Expansion of the surgical Apgar score across all surgical subspecialties as a means to predict postoperative mortality. Anesthesiology. 2011;114:1305-12.

18. Urrutia J, Valdes M, Zamora T, Canessa V, Briceno J. Can the Surgical Apgar Score predict morbidity and mortality in general orthopaedic surgery? Int Orthop. 2012;36:2571-6.

19. Ziewacz JE, Davis MC, Lau D, El-Sayed AM, Regenbogen SE, Sullivan SE, et al. Validation of the surgical Apgar score in a neurosurgical patient population. $J$ Neurosurg. 2013;118:270-9.

20. Khuri SF, Daley J, Henderson W, Barbour G, Lowry P, Irvin G, et al. The National Veterans Administration Surgical Risk Study: risk adjustment for the comparative assessment of the quality of surgical care. J Am Coll Surg. 1995;180:519-31.

21. Dindo D, Demartines N, Clavien PA. Classification of surgical complications; a new proposal with evaluation in a cohort of 6336 patients and results of a survey. Ann Surg. 2004;240:205-13.

22. Thorn CC, Chan M, Sinha N, Harrison RA. Utility of the Surgical Apgar Score in a district general hospital. World J Surg. 2012;36:1066-73.

23. Ferguson MK, Celauro AD, Prachand V. Prediction of major pulmonary complications after esophagectomy. Ann Thorac Surg. 2011;91:1494-500; discussion 1500-1.

24. Lagarde SM, Reitsma JB, Maris AK, van Berge Henegouwen MI, Busch OR, Obertop H, et al. Preoperative prediction of the occurrence and severity of complications after esophagectomy for cancer with use of a nomogram. Ann Thorac Surg. 2008;85:1938-45.

25. McCulloch P, Ward J, Tekkis PP, ASCOT group of surgeons; British OesophagoGastric Cancer Group. Mortality and morbidity in gastro-oesophageal cancer surgery: initial results of ASCOT mutlicentre prospective cohort study. BMJ. 2003; 327:1192-7.

26. Ferguson MK, Martin TR, Reeder LB, Olak J. Mortality after esophagectomy: risk factor analysis. World J Surg. 1997;21:599-603; discussion 603-4.

27. Lai F, Kwan TL, Yuen WC, Wai A, Siu YC, Shung E. Evaluation of various POSSUM models for predicting undergoing elective oesophagectomy for carcinoma. Br J Surg. 2007;94:1172-8.

28. Steyerberg EW, Neville BA, Koppert LB, Lemmens VE, Tilanus HW, Coebergh JW, et al. Surgical mortality in patients with esophageal cancer: development and validation of a simple risk score. J Clin Oncol. 2006;24:4277-84.

29. Ra J, Paulson EC, Kucharczuk J, Armstrong K, Wirtalla C, Rapaport-Kelz R, et al. Postoperative mortality after esophagectomy for cancer: development of a preoperative risk prediction model. Ann Surg Oncol. 2008;15:1577-84. Erratum in: Ann Surg Oncol. 2009;16:228.

30. Dutta S, Horgan PG, McMillan DC. POSSUM and its related models as predictors of postoperative mortality and morbidity in patients undergoing surgery for gastro-oesophageal cancer: a systematic review. World J Surg. 2010;34: 2076-82.

31. Grotenhuis BA, van Hagen P, Reitsma JB, Lagarde SM, Wijnhoven BP, van Berge Henegouwen MI, et al. Validation of a nomogram predicting complications after esophagectomy for cancer. Ann Thorac Surg. 2010;90:920-5.

32. Ferguson MK, Celauro AD, Prachand V. Assessment of a scoring system for predicting complications after esophagectomy. Dis Esophagus. 2011;24:510-5.

33. Monk TG, Saini V, Weldon BC, Sigl JC. Anesthetic management and one-year mortality after noncardiac surgery. Anesth Analg. 2005;100:4-10.

34. Zehetner J, DeMeester SR, Alicuben ET, Oh DS, Lipham JC, Hagen JA, et al. Intraoperative assessment of perfusion of the gastric graft and correlation with anastomotic leaks after esophagectomy. Ann Thorac Surg. 2015;262:74-8.

35. Blencowe NS, Strong S, McNair AG, Brookes ST, Crosby T, Griffin SM, et al. Reporting of short-term clinical outcomes after esophagectomy; a systematic review. Ann Surg. 2012;255:658-66.

Key Words: esophagectomy, esophageal carcinoma, risk prediction 